Serrano Manzano, B. \& De la Herrán Gascón, A. (2018). Creación de una red socioeducativa en espacios de exclusión social: una experiencia en el Distrito de Tetuán (Madrid). Revista Electrónica Interuniversitaria de Formación del Profesorado, 21(1), 1-16.

DOI: http://dx.doi.org/10.6018/reifop.21.1.263111

\title{
Creación de una red socioeducativa en espacios de exclusión social: una experiencia en el Distrito de Tetuán (Madrid)
}

\author{
Bianca Fiorella Serrano Manzano', Agustín de la Herrán Gascón ${ }^{2}$ \\ 'Universidad de Castilla la Mancha; \\ ${ }^{2}$ Universidad Autónoma de Madrid
}

\section{Resumen}

En este artículo se analiza la formación de una red socioeducativa en el Distrito de Tetuán (Madrid) en un contexto con notables índices de vulnerabilidad social. La organización surge desde un Plan de Desarrollo Comunitario, percibido como una oportunidad de mejora de la calidad de vida de la población. Con una orientación pedagógica, el objetivo principal es describir el proceso de creación de la estructura-red en un entorno de exclusión social, que pueda ser útil para aquellas comunidades que deseen transformarse en una red socioeducativa. Se emplea una metodología de corte cualitativo y etnográfico, implementando las siguientes técnicas para la recogida de información: observación participante, grupo de discusión, entrevista en profundidad y revisión de fuentes estadísticas y documentales relacionadas con el contexto. Los datos se analizan en dos momentos: en el análisis descriptivo y en el análisis de contenido. La muestra seleccionada consta de 52 personas procedentes de siete nacionalidades, que ocupan diversas posiciones en la configuración de la red. Como principales resultados, se caracteriza el contexto desde un diagnóstico comunitario y se definen siete dimensiones necesarias para la creación de una estructura socioeducativa en red. Por último, se identifican los actores, las fases y elementos clave que configuran su creación.

\section{Palabras clave}

Educación comunitaria; exclusión social; red socioeducativa; comunidad.

Contacto:

Bianca F. Serrano Manzano, bianca.serrano@uclm.es

Agustín de la Herrán, agustin.delaherran@uam.es 


\title{
Creation of a socio-educational network in areas of social exclusion: an experience in the District of Tetuán (Madrid)
}

\begin{abstract}
In this article, the creation of a social and educational network is analyzed, under circumstances of social vulnerability in Tetuán District (Madrid). This project springs from the Community Developing Plan which was considered as an opportunity for the improvement of the population living standard. From a pedagogic point of view, the main goal of this research is to describe the creation process of the network inside a social excluding environment and can be useful for those communities wishing to become a socio-educational network. A quality and ethnographic methodology has been followed, making use of the following data recollecting techniques: participating observation, discussion group, in-depth interview, information sources review and documentaries concerning the topic under study. The submitted data have been analyzed in two different ways: a descriptive analysis and a content analysis were carried out. The considered sample consists of 52 persons proceeding from seven different nationalities and who are in charge of different positions in the network configuration. As far as the main results of the research are concerned, the context is taken into account from a community assessment at first; seven dimensions (guidelines) are presented as necessary for the setting up of the social and educational structure of the network. In a second time, the social actors, the steps and the key-elements of the network creation are detailed.
\end{abstract}

\section{Key words}

Community education; social exclusion; socio-educational network; community.

\section{Introducción}

En este artículo se definen elementos, momentos y funciones clave en la creación de una red socioeducativa. Para Vilar (2009) el concepto red socioeducativa se puede entender desde diferentes perspectivas. Desde una perspectiva organizativa y geográfica se comprende como el mapa de recursos o servicios de un territorio. Desde un enfoque relacional, alude a la pertenencia a colectivos sociales o profesionales con los que se comparte información o se está conectado. Desde una posición comunitaria, enfatiza el trabajo para la creación de comunidad y tejido asociativo ("acción comunitaria", "animación comunitaria”, "trabajo comunitario"). Metodológicamente se refiere al modo cooperativo de concretar la acción en la realidad para construir un sistema entre profesionales y población, como respuesta a la complejidad social.

Esta investigación contempla estas cuatro perspectivas, deteniéndose principalmente en la tercera, analizando cómo se genera y organiza un determinado espacio socioeducativo con tendencia a la exclusión social y observando las interrelaciones que surgen espontáneamente a través de la acción comunitaria. El problema de esta investigación surge en un momento en el que las transformaciones socioeconómicas de los últimos lustros —desempleo, precariedad laboral, paro, envejecimiento, diversidad étnico-racial, cambio del modelo familiar, etc.- han producido y están produciendo nuevas formas de desigualdad. El término desigualdad adquiere relevancia en una sociedad que enfrenta, cada vez más, una grave crisis de cohesión. Para Subirats (2004) "el estudio de la 
desigualdad social ha estado tradicionalmente ligado al de la pobreza de forma casi exclusiva. Generalmente se ha relacionado a la pobreza con niveles bajos de ingresos, y habitualmente ésta se ha medido a través de la renta de las personas o los hogares" (p.13). Actualmente, no podemos basar el estudio de la desigualdad social únicamente en el factor económico ya que no suele ser el único elemento desencadenante. Desde la entrada a la sociedad de la información, nuevos mecanismos de segregación social han ido tomando forma. Durante la primera fase de la sociedad informacional, la dualización ha hecho crecer algunas desigualdades ya existentes en la sociedad industrial, pero a la vez, ha desarrollado otras más específicas de la nueva sociedad. Para Castells (1998) surgen procesos que afectan cada vez a más grupos de personas de nuevas y diferentes maneras: interactuantes e interactuados, es decir, quienes acceden, seleccionan y procesan la información disponible están siendo interactuados por quienes elaboran esa información, que son las actuales élites de la red. La sociedad de la información es desigual, básicamente, por sus exclusiones y discriminaciones en una red flexible en cambio continúo. En el ámbito educativo, siguiendo a Essombá (2014) el derecho a la igualdad educativa se ve reiteradamente vulnerado en la realidad cotidiana del alumnado de origen extranjero y de población en riesgo de exclusión social.

Actualmente, existen numerosos estudios en educación que demuestran que, cuando se separan a los grupos de personas (alumnado o familias) por niveles, ritmo, cultura, clase social etc. los participantes se distancian más (INCLUD-ED Consortium, 2009; Aubert, Flecha, García, Flecha \& Racionero, 2008). Lo mismo sucede en las grandes ciudades, dando lugar a guetos o espacios de degradación, poblados por colectivos en condiciones de vulnerabilidad social ${ }^{1}$ (Castells, 1997, 1998a, 1998b). Las políticas educativas que parten de una concepción que no promueven la inclusión de la diversidad a través de espacios de educación formal y no formal de calidad, están reproduciendo las desigualdades existentes y fomentado dinámicas exclusoras en el espacio urbano. En ese otro espacio que son las aulas, fenómenos como el bulling o el ciberbulling cobran cada vez mayor presencia, reflejando el interior de la escuela como un escenario de exclusión social y maltrato que demanda una intervención planificada (Cerezo \& Rubio, 2017). El contexto en el que se sitúa la investigación refleja esta coyuntura.

Para explicar la demanda de nuevas formas de intervención socioeducativa, se parte de una circunstancia y dinámica social que ha surgido con fuerza en los últimos tiempos bajo el término de exclusión social. Para Subirats (2004) la exclusión social es un fenómeno en constante cambio, estructural, multicausal y multidimensional que explica muchas situaciones de pobreza y desarraigo social.

Se han otorgado diferentes significados al vocablo exclusión social, lo que implica la circunscripción del término a un mayor número de categorías, acentuando aspectos como el desempleo, el trabajo precario y con escasa remuneración, la dificultad de acceso a la vivienda, la privación de acceso a recursos, la participación en instituciones públicas y la falta de disponibilidad de redes de interacción y participación social. Según INCLUD-ED Consortium (2009) "la exclusión social no se define sólo en términos de privación de ingresos, sino también como privación de capacidades básicas. La verdadera importancia de la exclusión social es hacer hincapié en el papel de las relaciones y de la experiencia de pobreza" (p. 6).

\footnotetext{
${ }^{1}$ En este trabajo se introduce el término vulnerabilidad social con el fin de no caer en el paradigma social en torno al concepto pobreza. Para Turner et al. (2003) el uso del concepto vulnerabilidad incluye tanto los riesgos, contingencias y presiones que puede experimentar una determinada población o grupo humano ante cualquier tipo de catástrofe, como la dificultad, indefensión o falta de medios por parte de dicho colectivo para afrontarla y reponerse ante ella
} 
En este sentido, Pérez Gómez (2012) afirma que "los ambientes desfavorecidos desde un punto de vista económico, social y cultural ofrecen a los estudiantes recursos educativos muy inferiores y estimulan actitudes y expectativas respecto a la escuela y el estudio mucho más pobres" (p. 79). En los territorios donde existen sectores vulnerables, la inversión e innovación en educación debería ser mayor, promoviendo que la escuela no se quede aislada del entorno. El centro educativo necesita incorporarse a proyectos compartidos con la comunidad. Esto no sucede en gran parte de las instituciones educativas y el fomento de la inclusión, continúa desarrollándose a través de actividades en pro de la igualdad en días concretos. Estas experiencias aisladas no tienen apenas repercusión en el proceso formativo (Díaz \& Anguita, 2017).

Siguiendo a Cívis \& Longás (2015) la emergencia de experiencias de trabajo en red, indica la búsqueda de respuestas a los retos socioeducativos que plantea una realidad social compleja, desigual, diversa y cambiante. En este sentido, Gordó i Aubarell (2010) señalan la importancia de tener en cuenta las siguientes premisas para crear espacios educativos en red:

1. La sociedad-red afecta por igual a empresas que a cualquier tipo de organización.

2. La red educativa no sólo plantea interacciones entre organizaciones, sino que también entran a formar parte de esta dinámica agentes individuales.

3. En el ámbito educativo esta estructura debe permitir romper con la competitividad entre las organizaciones e ir hacia una competencia de mayor alcance.

4. Conseguir la competencia de toda la red educativa obliga la interacción basada en términos de aprendizaje comunitario.

5. El carácter expansivo de la red la convierte en una potente estrategia de innovación.

La creación de espacios que promuevan la inclusión desde la heterogeneidad es un desafío para el sistema educativo. Sigue existiendo una fuerte tendencia generalizada, por parte del profesorado, alumnado y las propias familias, a entender la diversidad como acoger a "familias desestructuradas", inmigrantes, marginados sociales, pobres, etc. con efectos perjudiciales para su progenie (De la Rosa, 2017). Siguiendo a Cortina (2017), en las sociedades modernas surge un nuevo fenómeno, relacionado con lo anterior y que la autora acuña bajo el neologismo aporofobia. El término es pertinente en cuanto existe una expresión de la sociedad occidental acomodada en la que lo diverso molesta no solo por serlo, sino por ser pobre. De ellos es "la culpa de ser pobres" pues cuestionan nuestro estar- bien y nos inquietan con su pobreza, nos protegemos de ella y de la inseguridad que genera, rechazándolos e invisibilizándolos: "es el pobre el que molesta” (p.14).

Ante este contexto, el antídoto debe residir en la educación, en todos sus escenarios y ámbitos. Cabe destacar que antes de realizar un abordaje empírico se tomó como punto de partida, la revisión de diversas teorías que convergen en la epistemología que sustenta la creación de una red. Así pues, la investigación se fundamenta en gran medida en la Teoría de la Acción Comunicativa de Habermas y la Teoría de la Acción Dialógica de Freire. Ambos autores, salvando las diferencias, afirman a lo largo de sus obras que la razón no es exclusiva de nadie, sino que se construye dialógicamente con otro. El primero, planteó la necesidad de hacer nacer un nuevo paradigma denominado "giro lingüístico", manifestando que la interacción y más concretamente la comunicación y el lenguaje son elementos esenciales que construyen la realidad. En la teoría sociológica de Freire, no hay un "pienso", sino un "pensamos". Es el "pensamos" que establece el pienso y no al contrario. Esta coparticipación de los sujetos en el acto de pensar se da a través de la comunicación (Freire, 1973). 
Por otro lado, desde la Teoría Ecológica de Bronfenbrenner (1979) o el Pensamiento Ecológico de Bateson (1972) se rescata el concepto de sistema, como el conjunto de estructuras concéntricas que componen un ambiente ecológico y el cual influye en el desarrollo humano. Este desarrollo resultaría de la interacción recíproca entre el sujeto y el medio. En el ámbito educativo, estos sistemas pueden ser la familia, el colegio, el aula, el recreo, la ciudad, el barrio, etc. Desde este punto de vista, la red socioeducativa se definiría como un ambiente socio ecológico que interacciona con el sujeto, impactando positivamente en su evolución.

Es por ello que inmerso en este escenario demandante de nuevas estructuras educativas, el propósito general del presente estudio es describir el proceso de creación de una red socioeducativa en un contexto de exclusión social. Este objetivo se concreta en los siguientes específicos:

1. Caracterizar el entorno de exclusión social a través de un diagnóstico comunitario.

2. Determinar las principales dimensiones para la creación de una red.

3. Especificar las fases que la definen.

4. Analizar los principales elementos, actores y funciones en sus inicios.

\section{Metodología}

El campo de análisis empírico está determinado por la zona geográfica, el Distrito de Tetuán y las organizaciones, sujetos y procesos existentes, sobre los cuales se investiga. Dentro de este contexto se selecciona la creación de una red socioeducativa como fenómeno a observar y sobre el que se pretende obtener la información necesaria para describirlo.

El contexto de investigación se considera no sólo un espacio físico donde habita una determinada población, sino que es un espacio en el que se construyen dinámicas sociales, fruto de las interacciones, y en el que emergen dificultades y problemas, generándose respuestas a esas soluciones. En este sentido, la elección de este escenario para la investigación tiene que ver con la presencia de dos rasgos distintivos. Por un lado, la población del Distrito refleja, siguiendo a Gorz (1986) el modelo de social dual que ha ido definiendo la primera fase de la sociedad de la información (1973-1995). Las personas con habilidades académicas pueden acceder a los recursos y a la promoción laboral, mientras que los que carecen de esas habilidades se ven expuestos a una situación clara de exclusión social. Por otro lado, la existencia de amplio tejido asociativo en el entorno está en plena resonancia con la segunda fase de la sociedad de la información (a partir de 1995) definida por el objetivo de superar las desigualdades y dinámicas exclusoras producidas en la primera.

La presencia de población extranjera ha pasado a representar el 2,8\% de la población en 1996 a un 21, 98\% en 2007, provocando una resignificación de los espacios que definían el territorio antes de su llegada. Tetuán se configura, así, como el segundo Distrito de la capital con mayor número relativo de población de nacionalidad distinta a la española.

\section{Participantes}

El muestreo que se realiza es un muestreo teórico sin pretensiones estadísticas o de generalización de resultados. Los sujetos participantes están contemplados según los diferentes objetivos de la investigación y en función de estos se obtienen las características y la amplitud de la población a estudiar. 
Se contó para el estudio con un total de 52 personas ( 36 mujeres y 16 hombres) de siete orígenes diferentes (figura 1), con formación universitaria y no universitaria (figura 2). Las características heterogéneas de los participantes proporcionan rigurosidad, coherencia y validez a la investigación.

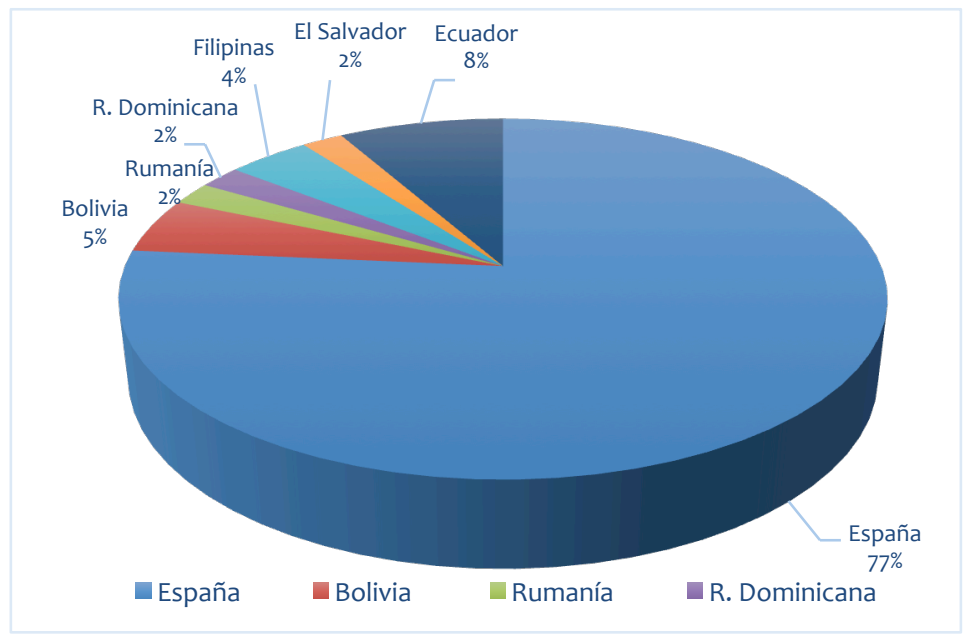

Figura 1. Origen de los participantes

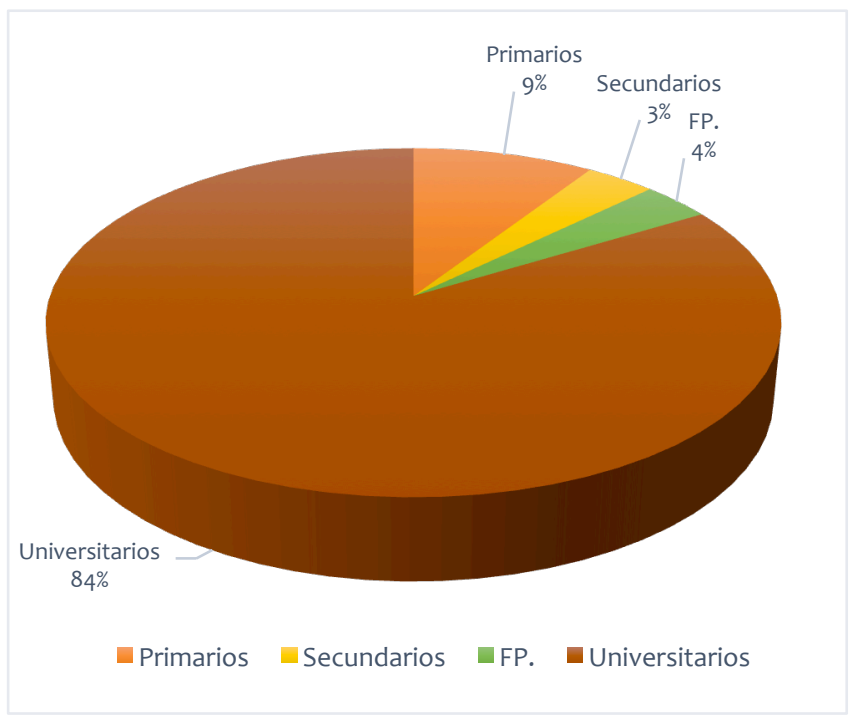

Figura 2. Formación de los participantes

\section{Diseño}

En cuanto a la metodología, el estudio se realiza desde un enfoque cualitativo, haciendo uso de la etnografía como estrategia. Siguiendo a Moral (2016) se diseña un estudio desde la perspectiva cualitativa ya que permite hacer el mundo visible a través de diferentes prácticas. El estudio se define como etnográfico, con el fin de describir las actividades de un grupo humano determinado, investigarla y recrear una realidad determinada. Como señalan Goetz y LeCompte (1988) permite una reconstrucción analítica de escenarios y grupos culturales intactos. Para Díez Gutiérrez (2013) el esquema etnográfico considera a la 
organización como un sistema cultural e intenta describirla y detectar sus problemas desde esta perspectiva.

\section{Instrumentos}

Lo habitual en el diseño etnográfico es acudir a estrategias de naturaleza fenomenológica ya que es la manera de sacar a la luz la producción subjetiva e intersubjetiva de conocimiento, desde la praxis de lo real y lo posible. Siguiendo a Goetz y Lecompte (1988) el diseño etnográfico requiere estrategias de investigación que conduzcan a la reconstrucción cultural. De esta manera, las técnicas seleccionadas para la obtención de datos han sido de corte cualitativo y son las siguientes: revisión documental, grupo de discusión, entrevista y observación participante.

\section{Procedimiento}

La investigación ha atravesado cinco fases:

- Profundización teórica, optando por compilar información relevante según tres unidades temáticas: exclusión/inclusión social, acción comunicativa (Habermas, 1987) y teoría dialógica (Freire, 1997a, 1997b). Desde este marco teórico se diseñaron los instrumentos para la recogida de la información. En esta etapa, adicionalmente, se entró en contacto con un grupo de investigación en la Comunidad de Madrid relacionado con el proyecto Comunidades de Aprendizaje (Elboj, Puigdellívol, Soller y Valls, 2002) y cuyos referentes teóricos eran afines a los de esta investigación.

- Definición de los objetivos/preguntas de investigación y de criterios para seleccionar la metodología.

- Selección de personas y colectivos participantes. En coherencia con el método de recogida de información, se trabajó con personas expertas (sujetos idóneos para el estudio) y sujetos-tipo donde el objetivo primordial fuera proporcionar al estudio, riqueza, profundidad y calidad de la información. Los participantes fueron agrupados de la siguiente forma: profesores, técnicos en educación, asociaciones, políticos y familias en situación de vulnerabilidad y niños/as.

- En la cuarta y quinta fases se recogieron los datos pertinentes y se realizaron los procesos progresivos de triangulación de fuentes, instrumentos e informes, asegurando la validez y pertinencia de la información elaborada. Se llevaron a cabo los contrastes necesarios hasta obtener una información que reflejara los significados y sentidos, consensos y disensos de las acciones y manifestaciones del fenómeno a analizar. Estas fases terminaron en un informe final que se remitió a la comunidad y a los expertos en el tema, que devolvieron el texto con anotaciones.

- Para la codificación de las transcripciones se utilizó el programa Atlas-TI, adoptando como criterio de división del discurso, las unidades de contenido temático según diferentes epígrafes o ejes temáticos. En opinión de Bartolomé (1981) la categorización hace referencia claramente, a los valores que la variable estudiada, en este caso la creación de una red socioeducativa, puede adoptar a través del análisis de contenido.

\section{Resultados}

A continuación, se presentan los resultados fruto del análisis cualitativo de la información obtenida. La codificación y reorganización de la información obtenida se sintetizó en cinco categorías que reúnen un grupo de elementos (unidades de registro) bajo el siguiente título: diagnóstico, dimensiones, actores, elementos y fases. Cada una de ellas representa 
un elemento explicativo, buscando acercar la comprensión de la estructura-red en el contexto descrito. Estas vertebran el análisis y se representan en la siguiente figura:

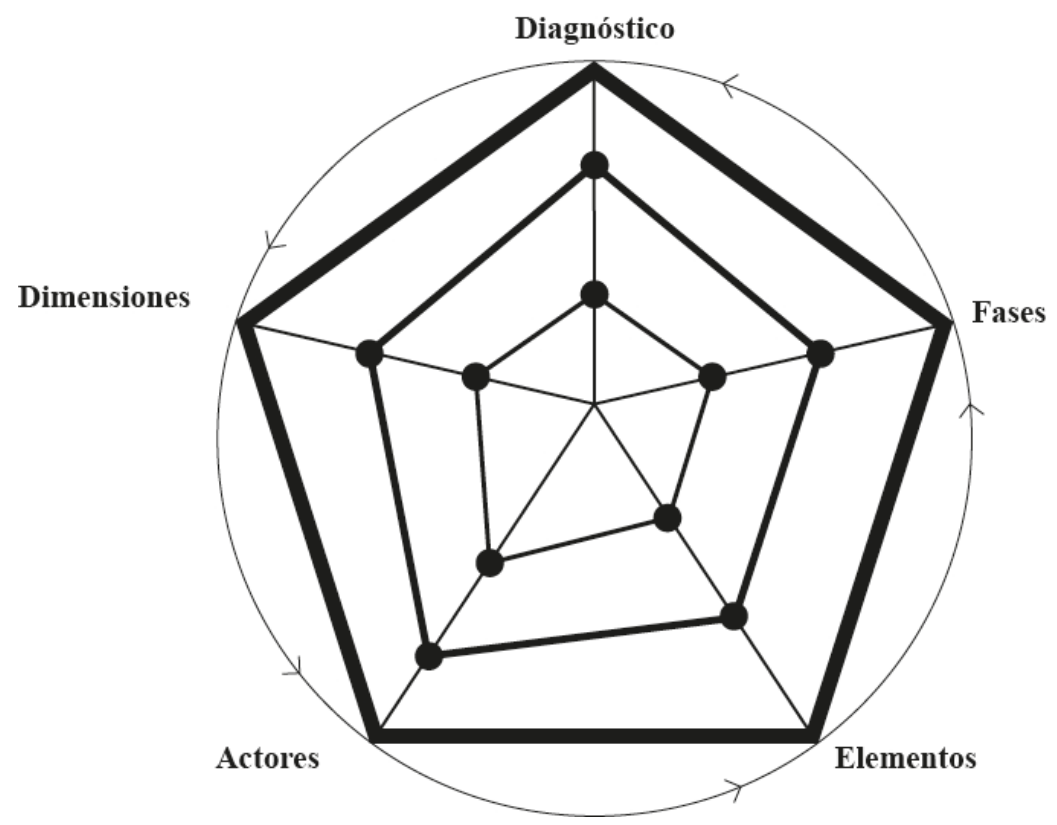

Figura 3. Categorías del análisis de resultados

\section{Diagnóstico}

En primer lugar, se caracteriza la situación y procesos de exclusión social vividos en el contexto de investigación. Se parte de esta categoría ya que se considera fundamental partir de un diagnóstico del contexto donde se sitúa la acción para después intervenir. De esta manera, las técnicas, metodologías y proceso de creación de la red se adaptarán a la realidad de cada comunidad y no al revés. Para ello, se recurre al análisis documental del diagnóstico participativo, enmarcado en el Proceso de Desarrollo Comunitario (PDC), realizado entre el año 2005 y 2006 por el equipo CIMAS $^{2}$ (Observatorio de Ciudadanía y Medio Ambiente Sostenible). En esta investigación participativa, se recogió la opinión propia de la población respecto a cómo observaban y vivían su entorno. Participaron alrededor de 1300 personas. Fruto de dicho estudio se identificaron cuatro nudos-críticos que, en el marco de esta investigación, validan y caracterizan el proceso de exclusión social que vive la comunidad, objeto de estudio: dinámica poblacional, entorno físico, servicios públicos y relaciones sociales. A continuación, se analizan los aspectos más relevantes del estudio, en cuanto a problemas y carencias vividos en el entorno como determinantes de dinámicas exclusoras.

Con respecto a la dinámica poblacional se constató una polarización manifiesta por pertenencia a dos comunidades: los "vecinos de toda la vida" y los "nuevos vecinos". Esta valoración surge a partir de la comparación entre un antes ("en que nos conocíamos

\footnotetext{
${ }^{2}$ Cabe aclarar que esta investigación parte de un estudio previo llevado a cabo en este contexto por CIMAS. "CIMAS somos profesionales del área de las ciencias sociales, personas formadas en distintas disciplinas comprometidas activamente con nuestro entorno social y político, en el que intervenimos con criterios de diversidad, inclusividad, creatividad y cooperación". Quienes Somos (s.f). En REDCIMAS. Recuperado el 07/06/2017. http://www.redcimas.org/pagina-ejemplo/hacemos/
} 
todos"), y un ahora ("los vecinos que han venido no los conocemos no sabemos nada de su vida, ni qué hacen"). La llegada de población inmigrante, procedente de diferentes nacionalidades, crea un paisaje heterogéneo y la percepción de inseguridad aparece vinculada a la aparición de grupos sociales por falta de contacto entre ellos. La percepción de la convivencia en la zona es negativa. La integración y convivencia intercultural entre comunidades se ve afectada por la percepción de los vecinos autóctonos de la inseguridad en el barrio en relación con la llegada de la población extranjera.

Para López (2005) uno de los elementos más característicos de la nueva configuración social consiste en el aislamiento de los sectores más ricos, que se traduce en la proliferación de los barrios privados y colegios, el aumento de las desigualdades y la crisis de la seguridad urbana. El contexto de estudio forma parte sin duda de está segmentación espacial existente en la Comunidad de Madrid.

\section{Dimensiones}

Una vez analizado el entorno en este apartado se identifican siete dimensiones, caracterizadas como condiciones previas, que se observaron para la creación de una red socioeducativa. Estas dimensiones podrían constituir los principios que guían la creación de la red socioeducativa y se definen brevemente a continuación:

$1^{\circ}$ ) Diversidad: inspira el trabajo en red, entendida como una oportunidad y no como un obstáculo que caracteriza a la sociedad actual y a la propia constitución de la red. Cuanto más heterogéneo sea el entorno en el que se circunscribe el trabajo comunitario, mayor es el reto educativo por construir estructuras que garanticen la igualdad, respetando que cada persona posee unas características únicas, especificidades, que le diferencian del otro. Dicha heterogeneidad la definen el conjunto de actores que se involucran en el contexto educativo, de diferente procedencia cultural, estatus social, capacidades cognitivas, etc.

$2^{\circ}$ ) Sentido: se refiere a que toda estructura o proceso educativo empieza y se modifica desde su sentido. El sentido está ligado a lo que acontece en la comunidad educativa, a cómo sucede y al valor atribuido a cada cosa, lo que depende de la cultura y el nivel de compromiso que se genere. Sólo podemos crear sentido en espacios de interacción en que se consensuen los temas o se generen conflictos fértiles o acuerdos. Trabajar por valores compartidos, teniendo en cuenta la expresión emocional de cada individuo, facilita el desarrollo comunitario y la experiencia socioeducativa en concreto.

$3^{\circ}$ ) Inclusión: enfatiza la importancia de que en el proceso de creación de la estructurared se garantice que las personas que presentan mayor vulnerabilidad social tengan las oportunidades y los recursos necesarios para empoderarse durante el proceso, acompañándoles en la participación para la toma de decisiones que afectan a sus vidas y el acceso a los derechos fundamentales. El desafío de la diversidad conlleva incluir a las personas en los procesos socioeducativos de manera efectiva.

$4^{\circ}$ ) Cooperación: conforma la dimensión central del proceso en el que se integran las tres dimensiones anteriores con las tres posteriores, incidiendo en cómo debe iniciarse el trabajo en grupo. Para que sea cooperativo los participantes deben compartir un mismo fin y alcanzar objetivos comunes (Johnson, Johnson y Holubec, 1999). Así se crea una interdependencia positiva de objetivos, recursos y roles, en la que cada uno necesita a los demás, interrelacionándose el éxito individual y grupal.

$5^{\circ}$ ) Comunicación dialógica: enfatiza la importancia del diálogo en el interior de la red y hacia fuera, con la comunidad. No todos los diálogos conducen a la comprensión entre las partes. Para ello los participantes deben llegar a acuerdos, desde pretensiones de 
validez (Habermas, 1987). "El diálogo es igualitario cuando se consideran las diferentes aportaciones en función de la validez de sus argumentos, en lugar de ser valoradas por las posiciones de poder de quienes las realizan" (Elboj y otros, 2002, p. 173). No sólo se produce a través de la palabra puede manifestarse a través de otras expresiones creativas: arte, música, teatro, etc.

$6^{\circ}$ ) Aprendizaje: se refiere a la dinámica principal que debe sostener la red. Las estructuras no desatienden el entorno: aprenden a vivir interactuando con él. Cada persona es afectada de modo significativo por las interacciones que se producen en el sistema. Como resultado de estas interacciones se sucederá un cambio cognitivo, moral y relacional a nivel individual y contextual.

$\left.7^{\circ}\right)$ Participación: es la respuesta coherente una vez ancladas las anteriores dimensiones. La estructura se abre a la comunidad insertándose en actividades educativas y políticas que fomenten la cohesión social. Las comunidades implicadas en proyectos de aprendizaje que integran intervenciones socioeducativas de diferentes agentes logran reducir significativamente las desigualdades y fomentan la inclusión social (INCLUD-ED Consortium, 2009). La organización que surge no se asienta en el control, miedo o desconfianza. Por el contrario, aparece una estructura en la que la conexión y participación para conseguir alta metas y expectativas educativas para todos son la clave.

\section{Actores}

Como ya se ha mencionado para la configuración de una red socioeducativa la riqueza y pluralidad de sujetos que forman parte de ella es un factor determinante. Además, la inclusión de grupos minoritarios o con menos poder y el diálogo y el intercambio entre los diferentes grupos de interés, constituye otro elemento esencial. En este epígrafe, se toma como definición de actor social la planteada por Touraine (1995) al afirmar que el actor social es todo aquel intenta realizar objetivos personales o colectivos porque está dentro de un entorno del cual es parte y por ello tiene muchas similitudes haciendo suyas la cultura y reglas de funcionamiento institucional, aunque solo sea parcialmente. Por tanto, el actor social no es entendido sólo como sujeto sino también como colectividad con intereses, condiciones y características que les definen como tales.

La multiplicidad de actores sociales (sectoralización) que influyen en el proceso de creación del sistema red, en la decisión, la ejecución o el control de las actividades públicas que diseñan, marcan la esencia misma de esta estructura multicéntrica. Por otro lado, para Longás (2009) la red debe ser capaz de integrar instituciones y actores en un plano de igualdad, unidos por el interés común, primero para compartir los análisis de las necesidades socioeducativas y los proyectos, y segundo para coordinar la acción de una manera integral y coherente.

Así mismo, y atendiendo a la máxima representatividad de los discursos existentes, se consideraron 4 grupos (stakeholders) contemplando la diversidad de sujetos implicados en el proceso comunitario de creación de red. A continuación, se nombran a los cuatro grupos de actores presentes en la creación de la red:

1. El actor político: Se refiere a todos aquellos sujetos que participan desde puestos de la Administración Pública y el Ayuntamiento y cuya capacidad de decisión sobre el entorno es muy elevada. Promueven el proceso de creación de la red desde el punto de vista institucional, lo financian económicamente y ceden espacios.

2. El actor técnico: Forman parte de este grupo, aquellos profesionales del ámbito socioeducativo que intervienen directamente con la población a través de diversas 
instituciones: Centro de Atención Primaria, de los servicios sociales y de los centros escolares. Dinamizan el proceso de creación.

3. El actor asociativo: Son todas aquellas personas integradas en una entidad social no lucrativa integrante de la plataforma de desarrollo comunitario. Suponen una "voz" acreditada y representativa del ámbito local, vinculada en este caso con el contexto socioeducativo.

4. El actor familia: No sólo hace referencia a los padres sino también a la participación de niños y niñas, puede incluirse las familias que participan en las AMPAS de los centros escolares u otros grupos de familias que se unen a través de estructuras informales. Se rompe el concepto de cliente o usuario del servicio educativo pasando a formar parte integrante de la red.

En el centro de la dinámica y en la que convergen algunos sujetos de los grupos de actores, se encuentra la Comisión de Educación. La creación de la Comisión supone el paso a una institución dinámica, planteando una nueva perspectiva de la educación, que cuenta con la participación de la población activa. Esta Comisión de Educación es entendida como el grupo motor de la red. Para Planas, Pineda, Gil y Sánchez (2014) "el grupo motor suele estar integrado por las personas de la comunidad que estén más motivadas para participar $e$ implicarse en la comunidad y tienen ganas de aprender".

El grupo motor se caracterizó por los siguientes rasgos: compromiso cívico, que se traduce en la participación de todos en los asuntos públicos; busca relaciones de igualdad, es decir relaciones horizontales de reciprocidad y cooperación, que dotan de un poder relacional en lugar de jerárquico; solidaridad, confianza y tolerancia entre sus miembros, lo que posibilita trabajar por objetivos comunes y apoyarse mutuamente, y asociacionismo civil que contribuye a la efectividad y estabilidad de la democracia (Bolívar, 2006).

La Comisión de Educación exigió para sus integrantes una formación específica y compleja, ya que, entre sus propósitos, figuró impulsar la reinvención del profesorado y del marco socializador de la escuela a través del asesoramiento y la conversión de las escuelas en espacios de apertura e integración del profesorado, familias y demás técnicos para trabajar en red. El grupo motor, constituido en esta red como Comisión de Educación dinamizó y colideró el proceso de creación de la red.

A modo de síntesis de este apartado, en la siguiente figura se incluye a los actores implicados en el proceso educativo y comunitario, como protagonistas del espacio-red:

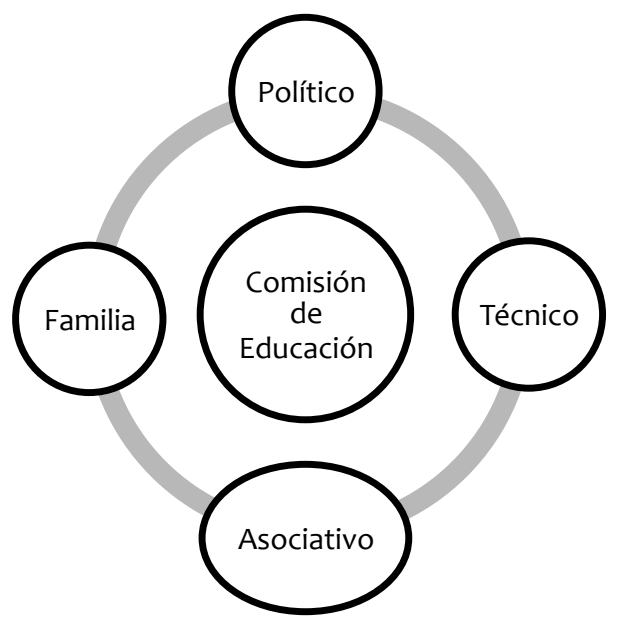

Figura 4. Actores red socioeducativa 


\section{Elementos}

Otro resultado relevante es la clasificación que se propone para diferenciar los elementos claves de la red en tres grupos: elementos cognoscitivos, estructurales y metodológicos. La definición de estos elementos se realiza de manera secuencial ya que unos necesitan de los otros:

$>$ En primer lugar, surgirían los elementos cognoscitivos. (Para qué) Predisponen el trabajo en red. Los principales elementos cognoscitivos son: las emociones, los valores, las actitudes, los límites y normas, las creencias que guiarán el trabajo. El lenguaje utilizado juega un papel fundamental para la construcción propia y conjunta sobre cómo se percibe realidad.

$>$ En segundo lugar, devienen los elementos estructurales (qué). Son los que facilitan el trabajo en red. Tienen que ver con la configuración y organización del espacio, con el diseño de estructuras (ambientes y escenarios) que garanticen la interacción. La propuesta estudiada exige una red interconectada en la que no haya linealidad comunicativa. El origen de una acción socioeducativa puede residir en diferentes ámbitos. Cualquier participante u organización puede promover una acción educativa y marcar la dinámica. Los espacios se crean en la dinámica generada por un permanente juego de correlaciones, sinergias e interacciones. Los elementos estructurales ayudan a: la toma de decisiones, la difusión de la información, la acción colectiva, los procedimientos y el intercambio de recursos etc.

$>$ Por último, se diferencian los elementos metodológicos (cómo). Comprendidos como el conjunto de acciones intencionales que favorecen el trabajo en red. Parten de presupuestos participativos y de un trabajo horizontal. Hacen referencia a cómo intervenir en la propia red, cómo dar forma a lo que se ha consensuado. La red se alimenta de acciones, interacciones, retroacciones y crece como un todo complejo.

Fases

Por último, se ha identificado siete fases en la génesis de la red que se describen seguidamente:

$1^{\circ}$ ) Sensibilización. En el Distrito de Tetuán se planteó iniciar un Plan de Desarrollo Comunitario a través del impulso de los técnicos en educación lo cual fue posible, por la existencia previa de un tejido asociativo que buscó dar respuesta a la situación de exclusión social y conflicto, vivida en el entorno.

$2^{\circ}$ ) Puesta en marcha. El inicio de la puesta en marcha del proceso de desarrollo comunitario estuvo acompañado por el asesoramiento de un investigador social. Desde su propuesta, se organizó al personal técnico del distrito mediante una estructura de coordinación mensual denominada Comité Técnico.

$3^{\circ}$ ) Diagnóstico comunitario. Se realizó con la ayuda de un equipo investigador desde una metodología participativa que implicó cuatro tipos de conversaciones: inicial, en red territorial, reflexiva y proyectiva. Estás interacciones fueron definidas por CIMAS (2006) como "una sucesión de espacios de conversación, en los que se da cuenta de múltiples realidades y se producen acuerdos entre las personas y los grupos".

$4^{\circ}$ ) Formación. Se encargó a un grupo especializado 3 llevar a cabo la formación y asesoramiento del equipo de desarrollo comunitario. Para ello se dividió al personal en cinco Comisiones Temáticas (Salud, Educación, Inmigración, Interculturalidad y

\footnotetext{
${ }^{3}$ Grupo liderado por Marco Marchioni, Trabajador e Investigador social en el ámbito del Desarrollo Comunitario.
} 
Discapacidad). Además, se constituyó un Grupo Motor, encargado de dinamizar el proceso por los seis barrios del distrito.

$5^{\circ}$ ) Activación desde un plan. A partir de las necesidades detectadas se empezó a trabajar en la elaboración de un Plan de Actuación Integral Sostenible (PAIS). Es una planificación estratégica a medio plazo que contempla la aportación ciudadana sobre las principales necesidades detectadas y la forma de resolverlas desde una perspectiva comunitaria y participativa.

$6^{\circ}$ ) Evaluación continua y formativa. La evaluación se ha realizado continuamente, en los distintos momentos del proceso de trabajo, los diferentes espacios y con los participantes de la comunidad educativa. Se utilizaron diversos instrumentos, previo consentimiento informado: cuestionarios cumplimentados por los estudiantes, grabación de foros y espacios virtuales, informes sobre contenidos, autoevaluaciones individuales y grupales e informes sobre las simulaciones de prácticas educativas.

$\left.7^{\circ}\right)$ Distanciamiento de la administración-acción social. La administración funcionó como aval, soporte o sostén al inicio, pero pasó a un segundo plano cuando la estructura comenzó a caminar independientemente.

\section{Discusión y conclusiones}

A la vista de los resultados expuestos, la creación de una red socioeducativa requiere de una serie de circunstancias, elementos y coyuntura específica que posibiliten el trabajo y lo diferencien de otras estructuras socioeducativas. Desde el análisis de buenas prácticas del trabajo en red realizado por Civís \& Longás (2015) se incide en la superación de los modos tradicionales de abordaje de las necesidades socioeducativas e indagar en nuevas formas que permitan dar respuesta a la complejidad de retos que debe asumir la educación hoy.

Según Villasante (1998, p. 48) "es necesario que exista voluntad política y asesoramiento experto o formación" para su creación. Esta idea concuerda con la investigación de Longás (2008) quien en su estudio afirma que: "en la mayoría de las experiencias exitosas estudiadas, el liderazgo ha correspondido a los entes locales -impulso político-técnico- y al mucho compromiso de los técnicos municipales de las áreas de atención a las personas" (p. 63). Además, siguiendo a Pastor (2009), la construcción de redes en los entornos comunitarios debe tratar de impulsar una forma de comunicación y cooperación horizontal en un territorio.

Para García \& Gómez (2009) las redes socioeducativas son en la actualidad un instrumento apropiado y de gran vigencia para llenar de sentido el modelo de desarrollo educativo y social. Se constata que una realidad compleja requiere actuaciones conjuntas que ayuden a proporcionar un sentido compartido y que comprometa a diferentes actores sociales. Las redes, desde su acción comunitaria horizontal, pueden convertirse en ese escenario colectivamente organizado donde cada ciudadano puede manifestar y desarrollar su concepto sobre la utilidad de las relaciones sociales, el diálogo y la vida en común.

Por otro lado, se concluye que el trabajo en red requiere un largo proceso de construcción a partir de la colaboración, la cooperación y el trabajo en equipo. En proyectos comparables, como el de Comunidades de Aprendizaje, Valls (1999) propone un desarrollo a lo largo de dos o tres años, según cada centro educativo. A diferencia del proyecto Comunidades de Aprendizaje los elementos de este estudio podrían aplicarse a la transformación de otros sistemas sociales y educativos, no sólo a un centro escolar: un ámbito territorial, un escenario local, un equipo de personas, un centro educativo, etc. 
Desde esta investigación, la cooperación se liga a la revitalización del tejido asociativo social para compartir la educación ciudadana en el ámbito de la familia y la ciudad (distritos y barrios). A su vez, la mayor autonomía de los centros y la tendencia a una mayor descentralización educativa de los municipios precisa realizarse con nuevas formas de participación que configuren "ciudades educadoras" (Luengo, 2006; Bolívar y Guarro, 2007).

El proceso resultante de las acciones anteriormente descritas debe estar presidido por dos grandes ideas: la participación de todos los agentes implicados (profesorado, alumnado, familias, agentes sociales, políticos etc.) generando un liderazgo compartido y la coherencia con los procesos y dinámicas que se tratan de construir: inclusión social, convivencia pacífica y cohesión (Luengo, 2006).

Para concluir, cabe señalar que teniendo en cuenta la propia naturaleza de la metodología planteada en la investigación y la dimensión singular del caso objeto de estudio, los resultados no se plantean como íntegramente generalizables. Sin embargo, la aplicación y/o contraste de estos resultados en trabajos similares en ámbitos educativos diversos, aportará comprensión sobre cómo ir diseñando y encaminando este tipo de innovaciones socioeducativas partiendo de las particularidades de cada contexto.

Con este trabajo se ha pretendido visibilizar que a pesar de las dificultades y la fragmentación social que se atraviesa en la actualidad, es posible y deseable diseñar espacios compartidos como el propuesto en esta investigación. Se ha mostrado que existe otra manera de establecer relaciones en base a la interacción, sobre principios dialógicos e igualitarios entre personas muy diversas y en entornos complejos que nos retan a innovar, emprender y proyectar nuevas estructuras educativas comprometidas con la transformación social.

\section{Referencias}

Aubert, A., Flecha, A., García, C., Flecha, R. \& Racionero, S. (2008). Aprendizaje dialógico en la sociedad de la información. Barcelona: Hipatia.

Bartolomé, M. (1981). Estudio crítico del método de análisis de contenido aplicado a la Investigación educativa. Documento policopiado. Barcelona: Universidad de Barcelona.

Bateson, Gregory (1972). Pasos hacia una ecología de la mente: colección de ensayos en antropología, psiquiatría, evolución y epistemología. Nueva York: Ballantine Books.

Bolívar, A. (2006). Familia y Escuela: Dos mundos llamados a trabajar en común. Revista de Educación (339), 119-146.

Bolívar A. \& Guarro, A. (2007). Educación y cultura democráticas: Proyecto Atlántida. Barcelona: Wolters Kluwer Educación.

Booth, T., Ainscow, M., Black-Hawhins, K., Vaughan, M. \& Shaw, L. (2000). The Index for inclusion: Developing learning and participation in schools. Bristol: Centre for Studies on Inclusive Education.

Brofenbrenner, U. (1979). The Ecology of Human Development: Experiments by Nature and Design. Cambridge: Harvard University Press. 
Castells, M. (1997). La Era de la información. Economía, sociedad y cultura. Vol.1. La sociedad red. Madrid: Alianza.

Castells, M. (1998a). La Era de la Información. Economía, sociedad y cultura. Vol. 2. El poder de la identidad. Madrid: Alianza.

Castells, M. (1998b). La era de la información. Economía, sociedad y cultura. Vol. 3. Fin del milenio. Madrid: Alianza.

Cerezo Ramírez, F. \& Rubio Hernández, F.J. (2017). Medidas relativas al acoso escolar y ciberacoso en la normativa autonómica española. Un estudio comparativo. Revista Electrónica Interuniversitaria de Formación del Profesorado 20(1), 113-126.

CIMAS (2006). Proceso de desarrollo comunitario Tetuán. Diagnóstico participativo. Resumen ejecutivo. Documents.mx. Recuperado de: http://documents.mx/documents/diagnostico-participativo-del-distrito-de-tetuanmadrid.html

Civís Zaragoza, M. \& Longás Mayayo, J. (2015). La colaboración interinstitucional como respuesta al desafío de la inclusión socioeducativa. Análisis de 4 experiencias de trabajo en red a nivel local en Cataluña. Educación XX1, 18(1), 213-236.

Cortina, A. (2017). Aporofobia. El rechazo al pobre. Barcelona: Paidós.

De la Rosa Moreno, L. (2017). Objetos convertidos en sujetos: encuentros con voces excluidas dentro de una asignatura sobre inclusión educativa. Revista Electrónica Interuniversitaria de Formación del Profesorado, 20(3), 209-223.

Díaz de Greñu Domingo, S. \& Anguita Martínez, R. (2017). Estereotipos del profesorado en torno al género y a la orientación sexual. Revista Electrónica Interuniversitaria de Formación del Profesorado, 20(1), 219-232.

Díez, E.J. (2013). Investigación-acción participativa: el cambio cultural con la implicación de los participantes. Revista Electrónica Interuniversitaria de Formación del Profesorado, 16(3), 137-153.

Elboj, C., Puigdellívol, I., Soler, M. \& Valls, R. (2002). Comunidades de aprendizaje. Transformar la educación. Barcelona: Graó.

Essombá, M.A (2014). Políticas de escolarización del alumnado de origen extranjero en el estado español hoy. Análisis y propuesta. Revista Electrónica Interuniversitaria de Formación del Profesorado, 17(2), 13-27.

Freire, P. (1997a). La educación en la ciudad. México: Siglo XXI.

Freire, P. (1997b). A la sombra de este árbol. Barcelona: El Roure.

García, J. y Gómez, J. (2009). Una experiencia de trabajo socioeducativo en red. Aula de Innovación Educativa (185), 70-74.

Goetz, J.P. y LeCompte, M.D. (1988). Etnografía y diseño cualitativo en investigación educativa. Madrid: Ediciones Morata.

Gordó i Aubarell, G. (2010). Centros educativos ¿Islas o Nodos? Barcelona: Graó.

Gorz, A. (1986). Los caminos del paraíso. Para comprender la crisis y salir de ella por la izquierda. Barcelona: Laia.

Habermas, J. (1987). Teoría de la acción comunicativa. I. Racionalidad de la acción y racionalización social. II. Crítica de la razón funcionalista. Madrid: Taurus. 
INCLUD-ED Consortium (2009). Actions for success in schools in Europe. Brussels: European Comission.

Johnson, D. W., Johnson, R. T. \& Holubec. E. J. (1999). El aprendizaje cooperativo en el aula. Buenos Aires: Paidós.

Longás, J. (2008). Redes socioeducativas y desarrollo comunitario. Introducción. Cultura y Educación, 20(3), 247-279.

Luengo, F. (2006). El Proyecto Atlántida: Experiencias para fortalecer el eje escuela, familia y municipio. Revista de Educación (339), 177-194.

Moral, C. (2016). Estrategias para resistir a la crisis de confianza en la investigación cualitativa actual. Educación XX1, 19(1), 159-177.

Pastor S. E. (2009). Participación y gestión de las políticas sociales municipales. Murcia: Editum.

Pérez Gómez, A. (2015). Educarse en la era digital. Madrid: Ediciones Morata.

Planas, A., Pineda-Herrero, P., Gil, E. y Sánchez, L. (2014). La metodología de la Evaluación Participativa de planes y acciones comunitarias. Tres experiencias de evaluación participativa en Catalunya. Pedagogía Social Revista Interuniversitaria (24), $105-134$.

Subirats, J. (2004). Pobreza y exclusión social. Un análisis de la realidad española y europea. Barcelona: Fundació la Caixa.

Touraine, A. (1995). Producción de la sociedad. México: UNAM.

Turner, B., Kasperson, R. E., Matson, P. A., McCarthy, J. J., Corell, R. W., Christensen, L., y otros. (2003). A framework for vulnerability analysis in sutainability science. Proceedings, National Academy of Sciences of the United States of America (14), 80748079 .

Valls, R. (1999). Comunidades de aprendizaje: una práctica educativa de aprendizaje dialógico para la sociedad de la información. Tesis doctoral. Universitat de Barcelona: Departament de Teoria i Història de l'Educació.

Vilar, J. (2009). Crear en la esfera local un mundo más humanizado y acogedor. El trabajo en red y la acción comunitaria. Monográficos Escuela (2), 4-6.

Villasante, T. (1998). De las redes sociales a las programaciones integrales. Buenos Aires: Lumen/Humanitas. 